\title{
The Relationship Between Excessive Alcohol Consumption and Alcohol Use Disorders According to DSM-IV and DSM-5
}

\author{
Marlous Tuithof, Margreet ten Have, Wim van den Brink, Wilma Vollebergh, and Ron de Graaf
}

\begin{abstract}
Background: Although it seems intuitive that alcohol use disorders (AUDs) include excessive alcohol consumption (EAC), this notion is not well established. This study investigates to which degree EAC (defined as $>14 / 21$ drinks weekly for women/men and at least three 5+ drinking days per week) and AUD overlap and whether problematic alcohol use groups (EAC-only, AUD-only, and $\mathrm{EAC}+\mathrm{AUD})$ differ from each other and from nonproblematic alcohol users regarding sociodemographics, mental health problems, functioning, and service utilization.

Methods: Data were derived from the Netherlands Mental Health Survey and Incidence Study-2, a population-based study including 5,443 current drinkers (aged 18 to 64) interviewed with the Composite International Diagnostic Interview 3.0. Both DSM-IV AUDs and a proxy of DSM-5 AUD are considered.

Results: Of the current drinkers, 3.8\% reported 12-month EAC. Twelve-month prevalence of DSM-IV and DSM-5 AUD were 5.4 and 4.4\%, respectively. Regarding DSM-IV, only 17.7\% of subjects with AUD reported EAC and $25.3 \%$ of those with EAC had an AUD. Compared with nonproblematic alcohol users, the 3 groups of problematic alcohol use (EAC-only, AUD-only, and EAC + AUD) were more often associated with mental health problems, poorer functioning, and service utilization. There were few differences between EAC-only and AUD-only regarding these correlates. However, EAC + AUD had strongest associations with above-mentioned correlates compared with the other 3 groups. Compared with DSM-IV findings, DSM-5 AUDs had slightly larger overlap with EAC, but correlates were similarly associated with problematic alcohol use groups.

Conclusions: Findings indicate limited overlap between EAC and AUD. Yet, both dimensions were similarly associated with other problems suggesting that both should be included in future epidemiological research to detect the total group of problematic alcohol users.
\end{abstract}

Key Words: Alcohol Consumption, Alcohol Use Disorder, DSM-IV, DSM-5, Classification.

$P$ SYCHIATRIC CLASSIFICATION SYSTEMS, such as the Diagnostic and Statistical Manual of Mental Disorders (DSM; American Psychiatric Association, 1994), describe alcohol use disorders (AUDs) as maladaptive patterns of alcohol use leading to significant impairment and distress. Excessive drinking, which can be distinguished into high average alcohol consumption and frequent heavy drinking days, is another dimension of problematic alcohol use. The 2 types of excessive drinking are each associated with serious health risks (World Health Organization, 2000) and

From the Netherlands Institute of Mental Health and Addiction ( $M T, M t H, R d G)$, Utrecht, the Netherlands; Department of Psychiatry $(W v d B)$, Academic Medical Centre, University of Amsterdam, Amsterdam; and Department of Interdisciplinary Social Science (WV), Utrecht University, Utrecht, the Netherlands.

Received for publication January 21, 2013; accepted June 5, 2013.

Reprint requests: Marlous Tuithof, MSc, Netherlands Institute of Mental Health and Addiction, PO Box 725, 3500 AS Utrecht, the Netherlands; Tel.: + 31 302971100; Fax: +31 302971111; E-mail: mtuithof@ trimbos.nl

Copyright $($ C 2013 by the Research Society on Alcoholism.

DOI: 10.1111/acer.12248 with alcohol-related problems (Caetano et al., 2012; Gmel et al., 2001). Co-occurrence of both types of excessive drinking may point to a small but serious group of alcohol users with a severe problematic drinking pattern in itself (Smith et al., 2010). In the remainder of this article, excessive alcohol consumption (EAC) will refer to this combination of excessive drinking types.

Although excessive drinking is not part of the AUD diagnosis, it could be argued that it is necessary for development of alcohol-related problems and therefore is an implicit characteristic of people with AUDs. However, this notion was not supported by findings from the first Netherlands Mental Health Survey and Incidence Study (NEMESIS; Verdurmen et al., 2003). Only one-third of the individuals with DSMIII-R alcohol abuse and half of those with alcohol dependence exceeded safe weekly drinking limits $(>14 / 21$ drinks weekly for women/men). Also, the other way around, only one-third of the drinkers exceeding these safe weekly limits met DSM-III-R criteria for an AUD. Other studies also observed a limited overlap between excessive drinking types and alcohol-related problems (Caetano et al., 2012; Gmel et al., 2001). Moreover, the overlap between AUD and excessive drinking could become even smaller than the 
overlap reported in previous studies if excessive drinking is defined as a combination of high average alcohol consumption and frequent heavy drinking days (i.e., EAC).

Examining the 2 dimensions of problematic alcohol use (EAC and AUD) in relation to each other is not only useful to gain insight into the degree of their overlap, but also to increase understanding regarding correlates of groups of problematic users; that is, excessive drinkers without alcohol-related problems (EAC-only), people with alcoholrelated problems without excessive drinking (AUD-only), and people with both aspects of problematic alcohol use (EAC + AUD). Many studies only use 1 dimension, but this has 2 important limitations. First, not all problematic alcohol users will then be included. For example, if only AUD is measured, excessive drinkers who do not meet DSM AUD criteria are overlooked, even though they might have similar problems in other areas of their life, for example, regarding mental health or functioning. Second, differences between AUD with or without excessive drinking cannot be detected, while mental health, functioning, and service utilization may be more severely affected in those with the combination of AUD and excessive drinking. Moreover, knowledge regarding associated sociodemographic characteristics may help targeting prevention at those at risk of more severe pathology.

Previous research provides some information regarding characteristics of the subgroups of problematic alcohol use. Sacco and colleagues (2009) observed that elderly people $(60+)$ with the combination of at-risk drinking and alcohol abuse-dependence symptoms more often had 12-month depression and poorer functioning than people with only at-risk drinking. However, both excessive drinking and AUDs are associated with younger age (Hasin et al., 2007; Karlamangla et al., 2006), and therefore, the findings of Sacco and colleagues (2009) cannot be generalized to the general population. Another study observed that rates of psychiatric disorders were higher among at-risk drinkers than among moderate drinkers or abstainers, but lower than among people with alcohol dependence (Bott et al., 2005). This study did not distinguish between dependence with and without atrisk drinking, but previous research suggested that comorbid psychiatric disorders may be stronger related to EAC than to symptoms caused by excessive consumption (Farrell et al., 2003). Conceivably, a gradient can be expected with people with AUD-only being least affected in other areas of their life, people with EAC + AUD being most affected and people with EAC-only being in between.

Using data from the second NEMESIS study (NEMESIS2), we aim to examine (i) to which degree EAC and AUD overlap; and if this overlap is limited, (ii) whether problematic alcohol use groups (EAC-only, AUD-only, and EAC + AUD) differ from each other and from nonproblematic alcohol users regarding various correlates, such as demographics, mental health, functioning, and service utilization. We expect that the 3 groups of problematic alcohol users are stronger associated with unfavorable outcomes than nonproblematic alcohol users (Bott et al., 2005; Sacco et al., 2009), that EAC-only is more strongly associated with unfavorable outcomes than AUD-only (Farrell et al., 2003), and that EAC + AUD has the strongest associations with negative outcomes (Sacco et al., 2009). To increase the power of our analyses, we combined DSM-IV abuse and dependence. In this paper, EAC is present if 2 types of excessive drinking co-occur, high average alcohol consumption (>14/21 drinks weekly for women/men) and frequent heavy drinking days (at least three 5+ drinking days per week). However, to increase our understanding of the overlap between EAC and AUD, we also looked at the overlap between separate excessive drinking types (high average alcohol consumption vs. frequent heavy drinking days) and separate AUDs (alcohol abuse vs. alcohol dependence). Because we are additionally interested in whether the relationship between EAC and AUD varies according to different DSM editions, we investigate our research questions separately for DSM-IV and a proxy of DSM-5 AUD.

\section{MATERIALS AND METHODS}

\section{Sample and Assessment Procedures}

NEMESIS-2 is a psychiatric epidemiologic survey in the Dutch general population. It is based on a multistage, stratified, random sampling of households, with 1 respondent randomly selected in each household. Data were collected between November 2007 and July 2009 (de Graaf et al., 2010). This resulted in a total sample of 6,646 adults aged 18 to 64 (response: $65.1 \%$ ). For the present analyses, those respondents who consumed at least 1 drink in the year preceding the interview were included $(n=5,443)$. The Composite International Diagnostic Interview (CIDI) 3.0 was used to determine the presence of EAC and of mental disorders. The CIDI is a fully structured, lay-administered interview developed by the World Health Organization, which is used worldwide. Clinical reappraisal interviews showed that it has generally good validity (Haro et al., 2006).

Alcohol Use Disorder. All respondents entered the alcohol section of the CIDI that assessed lifetime presence of symptoms of alcohol abuse, alcohol dependence, and craving. Moreover, respondents were asked whether symptoms of abuse or symptoms of dependence were still present in the past year and to rate impairment due to these symptoms. Computerized CIDI algorithms were used to generate 12-month DSM-IV abuse and dependence diagnoses. DSM-5 AUD symptoms include 3 of the 4 DSM-IV alcohol abuses (without legal problems) and all 7 DSMIV alcohol dependence criteria complemented with a new criterion covering craving. With 2 or more of 11 symptoms subjects meet criteria for AUD (American Psychiatric Association, 2013). All DSM-5 AUD symptoms were assessed using the CIDI 3.0 even though CIDI 3.0 was designed to yield DSM-IV diagnoses. Like DSM-IV, DSM-5 requires time clustering of symptoms, that is, the minimally required number of DSM-5 AUD symptoms must have occurred within the same 12-month period. In most cases, information regarding clustering of DSM-5 symptoms was not available as only clustering of 3 of 7 DSM-IV-dependence symptoms was assessed in the CIDI 3.0. This means that clustering of 2 or more alcohol abuse symptoms was not measured. Therefore, we could only construct a proxy of DSM5 AUD, using a symptom count without including a clustering criterion. 
Excessive Alcohol Consumption. Twelve-month EAC was present when respondents reported both high average alcohol consumption and frequent heavy drinking days. High average alcohol consumption was defined as drinking more than the international acknowledged safe drinking guidelines. Specifically, $>14$ drinks (standard drinks consisting of about $10 \mathrm{~g}$ of pure alcohol) weekly for women and $>21$ drinks weekly for men (British Medical Association, 1995; Haynes et al., 2005; Verdurmen et al., 2003). This was based on 2 questions: "In the past 12 months, how often did you usually have at least 1 drink-every day, nearly every day, 3 to 4 days a week, 1 to 2 days a week, 1 to 3 days a month, or less than once a month?" and "On the days you drank in the past 12 months, about how many drinks did you usually have per day?" Frequent heavy drinking days, defined as heavy volume drinking several times a week (Gmel et al., 2011; Li et al., 2007), was present if people consumed $5+$ drinks on at least 3 days a week. This was based on the question "How often in the past 12 months, did you have 5 or more drinks on a single day?" By this question, it was not possible to define different thresholds of a heavy drinking day for women and men.

Demographics. These included gender, age, educational level (4 categories: primary, basic vocational/lower secondary/higher secondary/higher professional, university), cohabitation status (living with a partner or not), employment status (in paid employment or not), and individual income (3 categories: low/middle/high).

Mental Health. The construction of DSM-IV mental disorder diagnoses with the CIDI 3.0 has been described in detail elsewhere (de Graaf et al., 2010). The following mental disorders were included in this study: mood (major depression, dysthymia, bipolar disorder), anxiety (panic disorder, agoraphobia, social phobia, specific phobia, generalized anxiety disorder), drug use (drug abuse and dependence), childhood disorder (attention-deficit/hyperactivity disorder, conduct disorder, oppositional-defiant disorder), and antisocial personality disorder. To increase accuracy of retrospective recall, childhood disorders were only assessed among respondents aged 18 to 44 (Kessler et al., 2007). Also, suicidal thoughts were included.

Functioning and Service Use. Functioning in the past month was based on the SF-36 (Stewart et al., 1988; Ware and Sherbourne, 1992). The 8 SF-36 scales were combined in 2 scales: physical functioning (general health, physical health, physical functioning, and bodily pain; $\alpha=0.78$ ) and mental functioning (psychological health, psychological functioning, social functioning, and vitality; $\alpha=0.78$ ), which ranged from 0 (poor) to 100 (good). Service use refers to 12 -month utilization of primary care, specialized mental health care, and addiction care for emotional or addiction problems.

\section{Data Analysis}

Analyses were performed using Stata, version 11.1 (Statacorp, 2009), which enabled us to control for the complex sampling and recruitment procedure of the study. The data were weighted to ensure they were representative of the national population. First, the overlap between EAC and AUD and the prevalence of problematic alcohol use groups were established (Tables 1-3). Multinomial logistic regression models were conducted to test group differences regarding demographics, mental health, functioning, and service use, adjusted for gender and age (Table 4). Stata produces relative risk ratios in multinomial regression analyses. According to Stata, these relative risk ratios should be interpreted as the risk of the particular group relative to the base group (Gutierrez, 2005), and they are thus very similar to odds ratios (ORs). To examine whether our definition of EAC influenced the results, sensitivity analyses were
Table 1. Twelve-Month Prevalence of Excessive Drinking Patterns and Alcohol Use Disorders (AUDs) Among 5,443 Current Drinkers in Unweighted Counts and Weighted Percentages

\begin{tabular}{lrr}
\hline & $n$ & $\%$ \\
\hline Excessive drinking patterns & & \\
High average alcohol consumption & 426 & 7.7 \\
Frequent heavy drinking days $^{\text {Excessive alcohol consumption }}{ }^{\mathrm{a}}$ & 328 & 6.4 \\
AUDs & 210 & 3.8 \\
DSM-IV & & \\
Alcohol abuse & & \\
Alcohol dependence & 190 & 4.5 \\
Any AUD & 35 & 0.9 \\
DSM-5 AUD & 225 & 5.4 \\
& 197 & 4.4
\end{tabular}

${ }^{a}$ Excessive alcohol consumption consists of high average alcohol consumption ( $>14 / 21$ drinks weekly for women/men) and frequent heavy drinking days (at least three $5+$ drinking days per week).

Table 2. The Overlap Between Excessive Drinking Patterns and AUD Among 5,443 Current Drinkers in Unweighted Counts and Weighted Percentages

\begin{tabular}{|c|c|c|c|c|}
\hline & \multicolumn{3}{|c|}{ DSM-IV AUD } & \multirow[t]{2}{*}{ DSM-5 AUD } \\
\hline & AA & $A D$ & AUD & \\
\hline \multicolumn{5}{|l|}{ HAAC } \\
\hline$\%$ of HAAC with reported disorder & 12.1 & 7.0 & 19.1 & 23.7 \\
\hline Unweighted $n$ & 40 & 23 & 63 & 83 \\
\hline$\%$ of reported disorder with HAAC & 20.6 & 60.9 & 27.3 & 41.8 \\
\hline \multicolumn{5}{|l|}{ FHDD } \\
\hline$\%$ of FHDD with reported disorder & 13.2 & 7.7 & 20.9 & 20.5 \\
\hline Unweighted $n$ & 33 & 20 & 53 & 61 \\
\hline$\%$ of reported disorder with FHDD & 18.7 & 55.0 & 24.7 & 29.9 \\
\hline \multicolumn{5}{|l|}{ EAC $^{\mathrm{a}}$} \\
\hline$\%$ of EAC with reported disorder & 12.3 & 12.8 & 25.1 & 29.2 \\
\hline Unweighted $n$ & 22 & 19 & 41 & 54 \\
\hline$\%$ of reported disorder with EAC & 10.4 & 54.7 & 17.7 & 25.3 \\
\hline
\end{tabular}

AUD, alcohol use disorder; AA, alcohol abuse; $A D$, alcohol dependence; HAAC, high average alcohol consumption; FHDD, frequent heavy drinking days; EAC, excessive alcohol consumption.

${ }^{a}$ EAC consists of HAAC ( $>14 / 21$ drinks weekly for women/men) and FHDD (at least three 5+ drinking days per week).

Table 3. Groups of Problematic Alcohol Use Among 5,443 Current Drinkers in Unweighted Counts and Weighted Percentages, Separately for DSM-IV and DSM-5 Alcohol Use Disorders (AUDs)

\begin{tabular}{lcrrrrrr}
\hline & & & \multicolumn{2}{c}{ DSM-IV } & & \multicolumn{2}{c}{ DSM-5 } \\
Group & EAC & AUD & \multicolumn{1}{c}{$n$} & \multicolumn{1}{c}{$\%$} & & \multicolumn{1}{c}{$n$} & $\%$ \\
\hline Group 1 & No & No & 5,049 & 91.7 & & 5,090 & 92.9 \\
Group 2 & Yes & No & 169 & 2.9 & & 156 & 2.7 \\
Group 3 & No & Yes & 184 & 4.5 & & 143 & 3.3 \\
Group 4 & Yes & Yes & 41 & 1.0 & & 54 & 1.1 \\
\hline
\end{tabular}

Presence of excessive alcohol consumption (EAC) refers to high average alcohol consumption (>14/21 drinks weekly for women/men) and frequent heavy drinking days (at least three $5+$ drinking days per week). Presence of DSM-IV AUD refers to alcohol abuse or dependence.

carried out with less stringent definitions of EAC; that is, frequent heavy drinking days or high average alcohol consumption; or one heavy drinking day per week and high average alcohol consumption. Level of significance was set at 0.05 . 


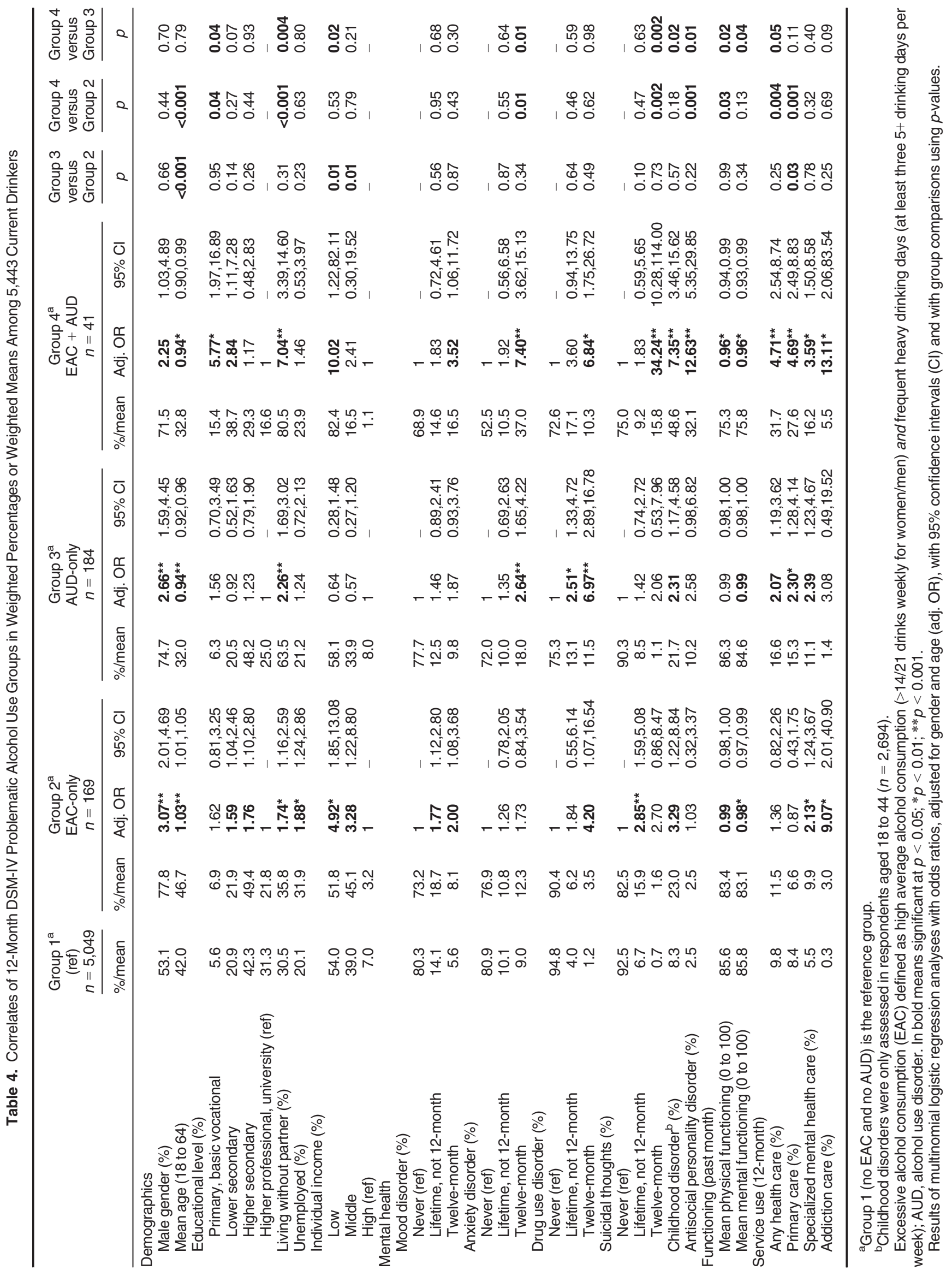




\section{RESULTS}

\section{Prevalence of EAC and AUD and Their Overlap}

Table 1 shows prevalence of excessive drinking patterns and AUDs. Of the total population, 7.7\% reported high average alcohol consumption, $6.4 \%$ frequent heavy drinking days, and $3.8 \%$ both types of excessive drinking, that is, EAC in the past year. Twelve-month DSM-IV alcohol abuse was present in $4.5 \%$ of the respondents and alcohol dependence in $0.9 \%$ (in total $5.4 \%$ reported a DSM-IV AUD). Twelve-Month DSM-5 AUD was about 20\% less prevalent than DSM-IV AUD: $4.4 \%$ reported a DSM-5 AUD.

As shown in Table 2, only 17.7\% of those with DSM-IV AUD reported EAC and $25.1 \%$ of subjects with EAC met criteria of DSM-IV AUD (Cohen's Kappa = 0.17). Notably, the proportion of EAC was considerably smaller for those with alcohol abuse $(10.4 \%)$ than for those with alcohol dependence (54.7\%). Compared with DSM-IV AUD, the overlap between EAC and DSM-5 AUD was slightly higher: $25.3 \%$ of those with DSM-5 AUD reported EAC and 29.2\% of subjects with EAC had a DSM-5 AUD (Cohen's Kappa $=0.24)$.

Four groups were created by combining EAC and AUD, separately for DSM-IV and DSM-5 (Table 3). The majority of the population belonged to the first group, including nonproblematic alcohol users with no EAC and no AUD (DSM-IV: 91.7\%; DSM-5: 92.9\%). Less than 3\% belonged to the second group (EAC-only). The third group (AUDonly) was larger for DSM-IV (4.5\%) than for DSM-5 $(3.3 \%)$. The fourth group, consisting of people with both dimensions of problematic alcohol use (EAC + AUD) was remarkably small (DSM-IV: 1.0\%; DSM-5: 1.1\%).

\section{Comparing DSM-IV Problematic Alcohol Use Groups}

Table 4 portrays frequencies, means, and results of multinomial regression analyses, adjusted for age and gender. First, the 3 problematic alcohol use groups were each compared with nonproblematic alcohol users; thus, no EAC and no DSM-IV AUD (group 1) was the reference group. The 3 problematic alcohol use groups were more often male and living without a partner. On average, people with EAC-only were older than nonproblematic alcohol users, whereas people with AUD-only and EAC + AUD were younger. Additionally, in comparison with nonproblematic alcohol users, EAC-only was more strongly associated with lower and higher secondary educational level than with the highest educational level (acting as the reference group), with being unemployed and with a lower income, whereas EAC + AUD was associated with low educational level and low income.

Compared with nonproblematic alcohol users, the 3 problematic alcohol use groups were each more often associated with clinical correlates. Specifically, EAC-only was associated with mood (lifetime and 12-month), drug use (12-month) and childhood disorder, suicidal thoughts (lifetime), poorer physical and mental functioning, utilization of specialized mental health care and of addiction care. AUDonly was associated with 12-month anxiety, drug use (lifetime and 12 month), childhood disorder, poorer mental functioning, utilization of any health care, primary care and specialized mental health care. EAC + AUD was associated with all 12-month mental disorders, childhood and antisocial personality disorder, poorer physical and mental functioning, and all types of service utilization.

Next, comparison of AUD-only and EAC-only (sixth column of Table 4) showed very few differences between these 2 groups. Particularly, people with EAC-only were older and had a lower income than people with AUD-only, whereas primary care utilization was higher in people with AUD-only. No further significant differences were observed.

Lastly, comparison of the groups with (EAC + AUD) and without (EAC-only, AUD-only) overlap (the last 2 columns of Table 4) showed that the associations with correlates were often strongest for the EAC + AUD group. Specifically, compared with EAC-only or AUD-only, EAC + AUD was more often associated with lower education, living without a partner, 12-month anxiety disorder, 12-month suicidal thoughts, antisocial personality disorder, poorer physical functioning, and utilization of any health care. Also, EAC + AUD more often had a low income, childhood disorder, and poorer mental functioning than AUD-only. Compared with EAC-only, EAC + AUD was more often associated with younger age and 12-month utilization of primary care.

\section{Comparing DSM-5 Problematic Alcohol Use Groups}

Results regarding DSM-5 groups differed slightly from DSM-IV findings, but the same picture emerged and the same conclusions can be drawn (table available on request). Specifically, problematic alcohol users were more often male, without a partner, more often had mental health problems, poorer physical and mental functioning, and service utilization than nonproblematic alcohol users. Like DSM-IV, comparison of DSM-5 AUD-only and EAC-only showed that the groups were quite similar regarding associations with correlates. However, contrasting DSM-IV findings, EAC + AUD no longer differed from EAC-only and AUDonly with regard to partner status and educational level. More importantly, the contrast between groups with overlap $(\mathrm{EAC}+\mathrm{AUD})$ and without overlap (EAC-only and AUDonly) was less outspoken in terms of mental health and functioning.

\section{Sensitivity Analyses}

Relaxing the EAC definition into frequent heavy drinking days or high average alcohol consumption; or into one heavy drinking day per week and high average alcohol consumption resulted in increase of the EAC + AUD group, but this group was still smaller than the AUD-only group. Moreover, the contrast between EAC + AUD and EAC-only or 
AUD-only became smaller and the 3 groups of problematic alcohol use became rather homogeneous. These results were the same for DSM-IV and DSM-5 AUDs (results are available on request).

\section{DISCUSSION}

Only a minority of the people with AUD also reported EAC in this population-based study, even though it seems intuitive that excessive drinking is needed for an AUD diagnosis. The problematic alcohol use groups (EAC-only, AUD-only, and EAC + AUD) were each associated with adverse outcomes in mental health, functioning, and service utilization. This suggests that a large problematic group of alcohol users with serious negative outcomes is overlooked if only 1 dimension is taken into account. Furthermore, co-occurrence of EAC and AUD was uncommon but was associated with most vulnerability. Targeted interventions should thus focus on this group. Characteristics that may help to identify people with EAC + AUD are lower educational level, living without a partner and low income.

\section{Limitations}

EAC was based on self-report, recall bias might be an issue then. Specifically, difficulties remembering the amounts and frequencies in an average week may have resulted in an underestimation of EAC. Thus, the groups with EAC-only and EAC + AUD could be somewhat larger in reality. Recall of lifetime mental disorders can also be a source of bias (Moffitt et al., 2010), resulting in underestimation of their prevalence rates, but possibly also in stronger associations with the problematic alcohol use groups.

It should be noted that prevalence rates of DSM-IV alcohol abuse and dependence in NEMESIS-2 were in accordance with other European observations, but were lower than prevalence rates observed in the United States and New Zealand (Grant et al., 2004; Kessler and Üstün, 2008). Also, the low prevalence of alcohol dependence $(0.9 \%)$ relative to abuse $(4.5 \%)$ differs from findings from the United States (Grant et al., 2004), where similar prevalence rates of alcohol abuse and dependence were observed. It is uncertain how these observations affect the generalizability of the present findings. If AUD diagnoses, and especially alcohol dependence, were somewhat more restricted in the present study, their overlap with alcohol consumption could even be smaller in countries or studies with higher AUD prevalence rates.

Due to the small number of cases with alcohol dependence, we had to combine DSM-IV alcohol abuse and dependence to study correlates of problematic alcohol use. When interpreting the results, it is important to keep in mind that the overlap between EAC and AUD was considerably smaller for abuse than for dependence and that abuse cases represent the majority of the AUD-only group, whereas abuse and dependence cases are more equally represented in the EAC + AUD group.
In our study, we focused on problematic alcohol use as portrayed by severe excessive drinking, not exceeding safe drinking guidelines. Therefore, our definition of EAC was stricter than those used in other studies (Sacco et al., 2009) or general drinking guidelines (Dawson and Grant, 2011). This means that only general comparisons are possible with other studies that focus on the relationship between exceeding safe drinking guidelines and alcohol-related problems (Caetano et al., 2012; Gmel et al., 2001; Sacco et al., 2009; Verdurmen et al., 2003).

DSM-5 requires that the necessary number of DSM-5 AUD symptoms must have occurred within the same 12-month period. However, as the CIDI 3.0 was designed to yield DSM-IV AUD diagnoses, information regarding clustering of symptoms was only available for 3 of the 7 DSM-IV dependence symptoms, not for 2 or more of the 11 DSM-5 AUD symptoms. Consequently, it was not possible to apply this clustering criterion in the DSM-5 diagnoses, and only a proxy of DSM-5 AUD using a symptom count could be constructed. Additional analyses showed that eliminating the clustering criterion in DSM-IV AUD increased the prevalence of alcohol dependence, but decreased the prevalence of alcohol abuse, resulting in unchanged overall AUD prevalence rates. We were not able to apply the clustering criterion for DSM-5, but we assume that this would have had a similar limited effect on DSM-5 prevalence rates.

\section{Findings}

A major finding of this study is that, even though it seems conceivable that considerable drinking is needed for an AUD diagnosis, only one-fifth of the subjects with DSM-IV AUD reported EAC. A limited overlap was also observed with less stringent definitions of EAC (Caetano et al., 2012; Gmel et al., 2001; Verdurmen et al., 2003) and for the proportion of EAC in both alcohol abuse and dependence cases. Yet, compared with DSM-IV AUD, a somewhat higher proportion of people with DSM-5 AUD reported EAC. This was not surprising as mild abuse cases with only 1 symptom are no longer diagnosed in DSM-5 (Agrawal et al., 2011). Conceivably, these mild cases were an important part of the DSM-IV AUD cases without co-occurring EAC. Nevertheless, although clinical research suggests that a persistent pattern of heavy drinking is needed to develop AUD (Storbjörk and Room, 2008), current results indicate that AUD diagnoses in population-based studies are more inclusive, that is, nonheavy drinkers also become diagnosed with AUD.

Partly, the limited overlap between EAC and AUD may be the result of an underestimation of alcohol consumption in the present study. Also, errors in the identification of AUD symptoms in population-based research could play a role. For example, Caetano (1999) observed that especially symptoms regarding impairment of control and tolerance are prone to misinterpretation and may lead to overestimation of AUD prevalence rates. Yet, the limited overlap 
between EAC and AUD may also reflect that they represent 2 separate dimensions of problematic alcohol use. Apparently, due to the difference in main focus, 1 dimension can present itself without the presence of the other.

Notably, comparison of the 3 problematic alcohol use groups (EAC-only, AUD-only, and EAC + AUD) with nonproblematic alcohol users showed that all problematic alcohol users more often reported mental health problems, poorer functioning, and service utilization than nonproblematic alcohol users. Thus, as expected, also the groups with only 1 aspect of problematic alcohol use reported serious problems in other areas of their life. This is in agreement with the literature about at-risk drinking and AUDs (Bott et al., 2005; Hasin et al., 2007; Teesson et al., 2010), and it implies that both dimensions should be taken into account to detect the total group of problematic alcohol users. This notion is further supported by comparison of EAC-only and AUD-only as these groups were very similar in their associations with mental health aspects. Yet, these findings also indicate that the association between psychiatric comorbidity and AUDs is not only due to alcohol consumption, but also to the AUD symptoms itself, thereby contradicting our hypothesis that EAC-only would be stronger associated with unfavorable outcomes than AUD-only (Farrell et al., 2003).

As expected, co-occurrence of EAC and AUD seemed to be associated with most vulnerability as it was more strongly associated with clinical correlates than EAC-only (Sacco et al., 2009) or AUD-only. An especially strong association was found with 12-month suicidal thoughts. Previous research observed a relation between suicide attempts and alcohol consumption (Kerr et al., 2011) and AUDs (Boenisch et al., 2010). Current findings additionally suggest that in people with both AUD and an excessive drinking pattern awareness for 12-month suicidal thoughts could be worthwhile. Moreover, the strong association between $\mathrm{EAC}+\mathrm{AUD}$ and 12-month mental disorders indicates that people with EAC + AUD should also be monitored for symptoms of other mental disorders (Kessler and Price, 1993; Merikangas et al., 1996).

Our results help to describe the problematic alcohol use groups with regard to sociodemographic aspects. Specifically, compared with nonproblematic alcohol users, AUD with and without EAC was more often associated with younger age (Grant et al., 2004; Hasin et al., 2007; Teesson et al., 2010), whereas EAC-only was more often associated with older age (Bott et al., 2005). This suggests that older people are better capable of maintaining an excessive drinking pattern without experiencing alcohol-related DSM problems, possibly because they created a living situation in which (excessive) alcohol consumption less often triggers alcoholrelated problems. The finding that EAC-only and especially EAC + AUD were related to lower educational level, and lower income is in line with a prospective study that suggested that lower educational level predicts excessive drinking (van Oers et al., 1999). This indicates that these factors may help to identify people at risk for severe problematic alcohol use.

\section{Implications}

The observed limited overlap between EAC and AUD indicates that excessive drinking and AUD diagnoses may measure 2 different aspects of problematic alcohol use in population-based research. Yet, as all problematic alcohol use groups had problems in other areas, combining the 2 dimensions can be worthwhile in public health research to detect the total group of problematic alcohol users. Moreover, people with the combination of EAC + AUD had the most severe problems in terms of psychiatric comorbidity and social functioning. It may be worthwhile to investigate whether escalation of problems can be prevented by special attention to people with 1 aspect of problematic alcohol use and existing correlates of EAC + AUD, for example, low socioeconomic status and living without a partner.

The current study used cross-sectional data, and it was therefore not possible to examine differences in the course of problematic alcohol use between the groups. It seems desirable to include both dimensions in future studies examining this course. Specifically, longitudinal epidemiological studies generally observe high remission rates of AUDs (de Bruijn et al., 2006), whereas clinical studies describe AUD as a chronic relapsing disorder (Storbjörk and Room, 2008). Perhaps, the higher rate of excessive drinking among those with AUD in clinical research compared with those in epidemiological research could play a role in this discrepancy (Dawson et al., 2008; Storbjörk and Room, 2008). Specifically, AUDs with excessive drinking may be associated with more persistency than AUDs without excessive drinking. Also, a substantial part of those who recover from AUD may still have EAC. This would imply that remission of AUD does not necessarily indicate remission of problematic alcohol use and its related health consequences. This should be examined in future longitudinal research.

\section{REFERENCES}

Agrawal A, Heath AC, Lynskey MT (2011) DSM-IV to DSM-5: the impact of proposed revisions on diagnosis of alcohol use disorders. Addiction 106:1935-1943.

American Psychiatric Association (1994) Diagnostic and Statistical Manual of Mental Disorders (DSM-IV). American Psychiatric Association, Washington, DC.

American Psychiatric Association (2013) Diagnostic and Statistical Manual of Mental Disorders (DSM-5). American Psychiatric Association, Washington, DC.

Boenisch S, Bramesfeld A, Mergl R, Havers I, Althaus D, Lehfeld H, Niklewski G, Hegerl U (2010) The role of alcohol use disorder and alcohol consumption in suicide attempts - a secondary analysis of 1921 suicide attempts. Eur Psychiatry 25:414 420.

Bott K, Meyer C, Rumpf HJ, Hapke U, John U (2005) Psychiatric disorders among at-risk consumers of alcohol in the general population. J Stud Alcohol 66:246-253. 
British Medical Association (1995) Alcohol Guidelines on Sensible Drinking. British Medical Association, London.

de Bruijn C, van den Brink W, de Graaf R, Vollebergh WAM (2006) The three year course of alcohol use disorders in the general population: DSMIV, ICD-10 and the Craving Withdrawal Model. Addiction 101:385-392.

Caetano R (1999) The identification of alcohol dependence criteria in the general population. Addiction 94:255-267.

Caetano R, Mills B, Pinsky I, Zaleski M, Laranjeira R (2012) The distribution of alcohol consumption and the prevention paradox in Brazil. Addiction 107:60-68.

Dawson DA, Grant BF (2011) The "gray area" of consumption between moderate and risk drinking. J Stud Alcohol Drugs 72:453-458.

Dawson DA, Stinson FS, Chou SP, Grant BF (2008) Three-year changes in adult risk drinking behavior in relation to the course of alcohol-use disorders. J Stud Alcohol Drugs 69:866-877.

Farrell M, Howes S, Bebbington P, Brugha T, Jenkins R, Lewis G, Marsden J, Taylor C, Meltzer H (2003) Nicotine, alcohol and drug dependence, and psychiatric comorbidity - results of a national household survey. Int Rev Psychiatry 15:50-56.

Gmel G, Kuntsche E, Rehm J (2011) Risky single-occasion drinking: bingeing is not bingeing. Addiction 106:1037-1045.

Gmel G, McKeganey N, Klingemann S, Müller R, Brenner D (2001) Revising the preventive paradox: the Swiss case. Addiction 96:273-284.

de Graaf R, ten Have M, van Dorsselaer S (2010) The Netherlands Mental Health Survey and Incidence Study-2 (NEMESIS-2): design and methods. Int J Methods Psychiatr Res 19:125-141.

Grant BF, Dawson DA, Stinson FS, Chou SP, Dufour MC, Pickering RP (2004) The 12-month prevalence and trends in DSM-IV alcohol abuse and dependence: United States, 1991-1992 and 2001-2002. Drug Alcohol Depend 74:223-234.

Gutierrez RG. Use of the term 'relative risk ratio' [Stata Web site]. April 21, 2005. Available at: http://www.stata.com/statalist/archive/. Accessed October 8, 2012.

Haro JM, Arbabzadeh-Bouchez S, Brugha TS, De Girolamo G, Guyer ME, Jin R, Lepine JP, Mazzi F, Reneses B, Vilagut G, Sampson NA, Kessler RC (2006) Concordance of the Composite International Diagnostic Interview Version 3.0 (CIDI 3.0) with standardized clinical assessments in the WHO World Mental Health Surveys. Int J Methods Psychiatr Res 15:167-180.

Hasin DS, Stinson FS, Ogburn E, Grant BF (2007) Prevalence, correlates, disability, and comorbidity of DSM-IV alcohol abuse and dependence in the United States: results from the National Epidemiologic Survey on Alcohol and Related Conditions. Arch Gen Psychiatry 64:830-842.

Haynes JC, Farrell M, Singleton N, Meltzer H, Araya R, Lewis G, Wiles NJ (2005) Alcohol consumption as a risk factor for anxiety and depression: results from the longitudinal follow-up of the National Psychiatric Morbidity Survey. Br J Psychiatry 187:544-551.

Karlamangla A, Zhou K, Reuben D, Greendale G, Moore A (2006) Longitudinal trajectories of heavy drinking in adults in the United States of America. Addiction 101:91-99.

Kerr WC, Subbaraman M, Ye Y (2011) Per capita alcohol consumption and suicide mortality in a panel of US states from 1950 to 2002. Drug Alcohol Rev 30:473-480.
Kessler RC, Angermeyer M, Anthony JC, DE Graaf R, Demyttenaere K, Gasquet I, DE Girolamo G, Gluzman S, Gureje O, Haro JM, Kawakami N, Karam A, Levinson D, Medina Mora ME, Oakley Browne MA, Posada-Villa J, Stein DJ, Adley Tsang CH, Aguilar-Gaxiola S, Alonso J, Lee S, Heeringa S, Pennell BE, Berglund P, Gruber MJ, Petukhova M, Chatterji S, Ustün TB (2007) Lifetime prevalence and age-of-onset distributions of mental disorders in the World Health Organization's World Mental Health Survey Initiative. World Psychiatry $6: 168-176$.

Kessler RC, Price RH (1993) Primary prevention of secondary disorders: a proposal and agenda. Am J Community Psychol 21:607-633.

Kessler RC, Üstün TB (2008) The WHO World Mental Health Surveys. Global Perspectives on the Epidemiology of Mental Disorders. Cambridge University Press, New York, NY.

Li TK, Hewitt BG, Grant BF (2007) The Alcohol Dependence Syndrome, 30 years later: a commentary. The 2006 H. David Archibald lecture. Addiction 102:1522-1530.

Merikangas KR, Angst J, Eaton W, Canino G (1996) Comorbidity and boundaries of affective disorders with anxiety disorders and substance misuse: results of an international task force. Br J Psychiatry 168:58-67.

Moffitt TE, Caspi A, Taylor A, Kokaua J, Milne BJ, Polanczyk G, Poulton R (2010) How common are common mental disorders? Evidence that lifetime prevalence rates are doubled by prospective versus retrospective ascertainment. Psychol Med 40:899-909.

van Oers JA, Bongers IM, van de Goor LA, Garretsen HF (1999) Alcohol consumption, alcohol-related problems, problem drinking, and socioeconomic status. Alcohol Alcohol 34:78-88.

Sacco P, Bucholz KK, Spitznagel EL (2009) Alcohol use among older adults in the National Epidemiologic Survey on alcohol and related conditions: a latent class analysis. J Stud Alcohol Drugs 70:829-838.

Smith GW, Shevlin M, Murphy J, Houston JE (2010) An assessment of the demographic and clinical correlates of the dimensions of alcohol use behaviour. Alcohol Alcohol 45:563-572.

Statacorp (2009) Statacorp. Stata Statistical Software: Release 11. Statacorp LP, College Station, TX.

Stewart AL, Hays RD, Ware JE (1988) The MOS short-form general health survey: reliability and validity in a patient population. Med Care 26:724735.

Storbjörk J, Room R (2008) The two worlds of alcohol problems: who is in treatment and who is not? Addict Res Theory 16:67-84.

Teesson M, Hall W, Slade T, Mills K, Grove R, Mewton L, Baillie A, Haber P (2010) Prevalence and correlates of DSM-IV alcohol abuse and dependence in Australia: findings of the 2007 National Survey of Mental Health and Wellbeing. Addiction 105:2085-2094.

Verdurmen J, Monshouwer K, van Dorsselaer S, de Graaf R (2003) Bovenmatig Drinken in Nederland (Excessive Drinking in the Netherlands). Trimbos-instituut, Utrecht.

Ware JE, Sherbourne CD (1992) The MOS 36-item short-form health survey (SF-36): I. Conceptual framework and item selection. Med Care 30:473-483.

World Health Organization (2000) International Guide for Monitoring Alcohol Consumption and Related Harm. World Health Organization, Geneva. 